\title{
ON DERIVED CATEGORIES AND DERIVED FUNCTORS
}

\author{
SAMSON SANEBLIDZE
}

\begin{abstract}
For an abelian category, a category equivalent to its derived category is constructed by means of specific projective (injective) multicomplexes,
\end{abstract} the so-called homological resolutions.

\section{INTRODUCTION}

The derived category $D(\mathcal{A})$ of an abelian category $\mathcal{A}$ was introduced by Verdier in 1963, see [15] and [16. It was defined as the localization of the category of unbounded chain complexes with respect to quasi-isomorphisms. The existence of $D(\mathcal{A})$ creates set-theoretical problems. Verdier proved the existence of $D(\mathcal{A})$ only in the case when $\mathcal{A}$ has finite global dimension. Later the existence of $D(\mathcal{A})$ was established by Spaltenstein [14 in the case when $\mathcal{A}$ is the category of modules over a ring, or, more generally, the category of modules over a sheaf of rings. The first case was also considered by Hovey in $[\underline{8}$. Recently the existence of $D(\mathcal{A})$ was proved in the case when $\mathcal{A}$ is a Grothendieck category, see for example [1].

In the present paper we prove the existence of $D(\mathcal{A})$ in the case when $\mathcal{A}$ has enough projectives and countable coproducts. By duality the same is true provided $\mathcal{A}$ has enough injectives and countable products.

The essential part of the paper was in fact written about 15 years ago when the author was visiting the Heidelberg University. As it is partially reviewed above in the meantime there appeared various kinds of descriptions of derived categories, however, the decision to write this paper is motivated by reasons mentioned above and continued below: Our approach uses a theory of multicomplexes and emphasis a role of the homology of differential graded objects (bounded or unbounded) on the additive level; nowadays multicomplexes are considered to be endowed with multiplicative or higher order operations that measure certain standard relations up to homotopy (see, for example, [6, [9, [12, 13]). It should be noted that such enriched homological multicomplexes are candidates to be (co)fibrant objects in the appropriated closed model category, since the analogs of Proposition 2 below (compare Proposition 3 in [13). So that it is expected to use them for homotopy classification problems behind the rational homotopy theory too.

I thank T. Pirashvili for useful discussions. I also thank J. Huebschmann for comments.

Key words and phrases. derived category, derived functor, multicomplex, homological resolution. 


\section{The MAIN RESULT}

A chain map between unbounded chain complexes which induces an isomorphism in homology (i.e. a quasi-isomorphism) is not a homotopy equivalence even each complex consists of projective objects of an abelian category $\mathcal{A}$, and also an additive functor, such as $\mathrm{Hom}$ and $\otimes$ one, does not preserve quasi-isomorphisms 4]. There are various kinds of restrictions on chain complexes that guarantees quasi-isomorphisms to be homotopy equivalences (see [4, [14, 8], 5]).

Here we consider the other kind of restriction by introducing special projective (injective) chain complexes, homological multicomplexes. In particular, by means of these complexes we can describe the derived category $D(\mathcal{A})$ and to construct the derived functor for an additive functor mentioned in the introduction.

Usually the above restrictions are evoked to start inductively from the first nontrivial component of a (bounded) chain complex. In our case, the induction relies on a specific filtration of a multicomplex involving all (total) degrees simultaneously (compare [4, 8]). On the other hand, given a chain complex $A$ over $\mathcal{A}$, one considers in the theory of derived category projective (injective) substitutions $C \rightarrow A(A \rightarrow C)$ of $A$, i.e. quasi-isomorphisms with $C$ consisting of projective (injective) components from $\mathcal{A}$. We show that for each chain complex $A$ there is a multicomplex $C$ such that its total complex is quasi-isomorphic to $A$ and $C^{*, j}$ is a projective resolution of the cohomology $H^{j}(A)$ for each $j \in \mathbb{Z}$. So that among projective substitutions of $A$ mentioned above, the homological resolution $C$ could be chosen small as possible.

In order to state our main theorem below we choose the language of projective objects (the case of injective objects is entirely dual).

Given an abelian category $\mathcal{A}$, a multicomplex over $\mathcal{A}$ is a bigraded object $C^{*, *}=$ $\left\{C^{i, j}\right\}_{i, j \in \mathbb{Z}}$ together with morphisms $d^{r}: C^{i, j} \rightarrow C^{i+r, j-r+1}, r \geq 0$, such that $\sum_{p+q=n} d^{p} d^{q}=0$ for each $n \geq 0$. The total complex of $C^{*, *}$ is the chain complex $\left(\operatorname{Tot}(C), d^{*}\right)$ with

$$
\operatorname{Tot}(C)^{n}=\bigoplus_{i+j=n} C^{i, j} \text { and } d^{*}=d^{0}+d^{1}+\cdots+d^{r}+\cdots .
$$

In particular, $d^{1} d^{1}=0$ when $d^{0}=0$. A multicomplex $\left(C^{*, *}, d^{*}\right)$ is called homological if $d^{0}=0, C^{i, *}=0$ for $i>0$ and $H^{i}\left(C^{i, *}, d^{1}\right)=0$ for $i<0$. A multicomplex $\left(C^{*, *}, d^{*}\right)$ is called projective if each $C^{i, j}$ is a projective object of $\mathcal{A}$. A column (resolution) filtration of a multicomplex $\left(C^{*, *}, d^{*}\right)$ is a sequence $\left\{C_{(k)}\right\}_{k \leq 0}$ with $C_{(k)}=\bigoplus_{k \leq i \leq 0} C^{i, *}$.

A multicomplex map $f: A \rightarrow B$ between two multicomplexes $A$ and $B$ is a chain map of total degree zero that preserves the column (resolution) filtration, i.e. $f \operatorname{Tot}(A)^{n} \subset \operatorname{Tot}(B)^{n}$ and $f A_{(k)} \subset B_{(k)}$; so that $f$ has the components $f=f^{0}+$ $\cdots+f^{i}+\cdots$ with $f^{i}: A^{s, t} \rightarrow B^{s+i, t-i}$. A homotopy between two maps $f, g: A \rightarrow B$ of multicomplexes is a chain homotopy $s: A \rightarrow B$ of total degree -1 that lowers the column filtration by 1, i.e. $\operatorname{sTot}(A)^{n} \subset \operatorname{Tot}(B)^{n-1}$ and $s A_{(k)} \subset B_{(k-1)}$.

Note that, unlike standard bicomplexes, in a homological multicomplex we have no vertical differentials; this fact together with the acyclicity with respect to the horizontal differential $d^{1}$ guarantees the spectral sequence arising from the column filtration to be collapsed; in particular, the other components $d^{r}, r \geq 2$, have no action to change the cohomology non-isomorphically; in other words, when $d^{r}$ varies 
in $\operatorname{Hom}\left(C^{i, j}, C^{i+r, j-r+1}\right)$ for $r \geq 2$ one obtains multicomplexes with isomorphic cohomologies (see Fig. 1).

Let $K(\mathcal{A})$ be the category whose objects are chain complexes over $\mathcal{A}$ and morphisms are homotopy classes of maps denoted by $[-,-]$, while $K_{\mathcal{P}}(\mathcal{A})$ be the category whose objects are homological projective multicomplexes over $\mathcal{A}$ and morphisms are homotopy classes of maps denoted by $[-,-]_{\mathcal{P}}$.

Recall that the derived category $D(\mathcal{A})$ of $\mathcal{A}$ is isomorphic to the category obtained from $K(\mathcal{A})$ by inverting the class of quasi-isomorphisms [5], [10], [15], and let $Q: K(\mathcal{A}) \rightarrow D(\mathcal{A})$ be the localization functor. Let $\kappa: K_{\mathcal{P}}(\mathcal{A}) \rightarrow K(\mathcal{A})$ be the functor defined by the following obvious proposition.

Proposition 1. Given a multicomlex $C$ and a morphism $\mathfrak{f}: C \rightarrow C^{\prime}$ in $K_{\mathcal{P}}(\mathcal{A})$, the assignments $C \rightarrow \operatorname{Tot}(C)$ and $\mathfrak{f} \rightarrow[\operatorname{Tot}(f)]$ define a functor $\kappa: K_{\mathcal{P}}(\mathcal{A}) \rightarrow K(\mathcal{A})$ for a representative $f$ of $\mathfrak{f}$.

Proof. First remark that the assignments $C \rightarrow \operatorname{Tot}(C)$ and $f \rightarrow \operatorname{Tot}(f)$ define a functor from the category of multicomplexes and multicomplex maps to the category of chain complexes and chain maps over $\mathcal{A}$. Now if $f, g: C \rightarrow C^{\prime}$ are two chain homotopic maps of multicomplexes $f \underset{s}{\simeq}$, then clearly $s$ induces a map Tot $(s)$ : $\operatorname{Tot}(C) \rightarrow \operatorname{Tot}\left(C^{\prime}\right)$ such that $\operatorname{Tot}(f) \underset{\operatorname{Tot}(s)}{\stackrel{\sim}{\sim}} \operatorname{Tot}(g)$.

Consider the functor

$$
\iota: K_{\mathcal{P}}(\mathcal{A}) \rightarrow D(\mathcal{A})
$$

obtained as the composition $K_{\mathcal{P}}(\mathcal{A}) \stackrel{\kappa}{\rightarrow} K(\mathcal{A}) \stackrel{Q}{\rightarrow} D(\mathcal{A})$.

The main statement here is the following

Theorem 1. If an abelian category $\mathcal{A}$ has enough projectives and countable coproducts, then the functor $\iota: K_{\mathcal{P}}(\mathcal{A}) \rightarrow D(\mathcal{A})$ is an equivalence of categories.

This theorem relies on the following 'Whitehead (or Adams-Hilton) type' proposition that has an independent interest.

Proposition 2. Given a homological projective multicomplex $C$ and a quasi-isomorphism $f: A \rightarrow B$ in $K(\mathcal{A})$, the induced map $f_{\#}:[\operatorname{Tot}(C), A] \rightarrow[\operatorname{Tot}(C), B]$ is a bijection.

\section{Proof of Theorem 1}

Given a chain complex $(A, d)$ from $K(\mathcal{A})$, its homological resolution is a homological projective multicomlex $\left(C^{*, *}, d^{*}\right)$ with a quasi-isomorphism $\phi:\left(\operatorname{Tot}(C), d^{*}\right) \rightarrow$ $(A, d)$. In particular, $\left(C^{*, j}, d^{1}\right)$ forms a projective resolution of the object $H^{j}(A)$ for all $j \in \mathbb{Z}$ (see Fig. 1). 


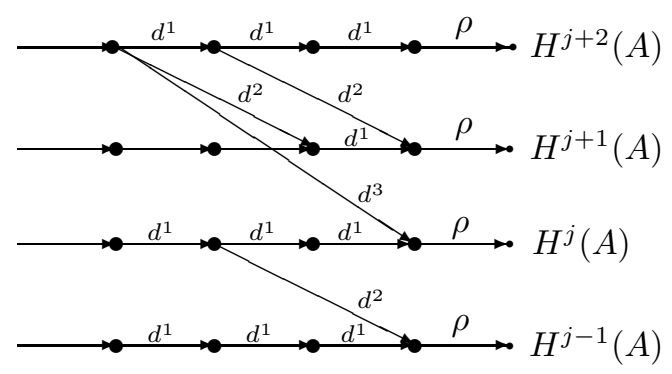

$$
\cdots \rightarrow C^{-3, *} \stackrel{d^{1}}{\rightarrow} C^{-2, *} \stackrel{d^{1}}{\rightarrow} C^{-1, *} \stackrel{d^{1}}{\rightarrow} C^{0, *} \stackrel{\rho}{\rightarrow} H^{*}(A)
$$

Figure 1. A fragment of a homological resolution.

Proposition 3. If an abelian category $\mathcal{A}$ has enough projectives and countable coproducts, then any chain complex $A$ of $K(\mathcal{A})$ has a homological resolution $\phi$ : $\operatorname{Tot}(C) \rightarrow A$.

Proof. First choose a projective resolution $\rho:\left(C^{*, j}, d^{1}\right) \rightarrow H^{j}(A)$ of $H^{j}(A)$ for each $j \in \mathbb{Z}$ so that $d^{1}: C^{i-1, *} \rightarrow C^{i, *}$ for $i \leq 0$. Consider the epimorphism

$$
\rho^{0}=\left.\rho\right|_{C^{0, *}}: C^{0, *} \rightarrow H^{*}(A) .
$$

Factor it through cocycles $C^{0, *} \stackrel{\phi^{\prime}}{\rightarrow} Z A^{*} \stackrel{\nu}{\rightarrow} H^{*}(A)$ and obtain a morphism $\phi^{0}$ : $C^{0, *} \stackrel{\phi^{\prime}}{\rightarrow} Z A^{*} \hookrightarrow A^{*}$. Define also a morphism

$$
\phi^{1}: C^{-1, *} \rightarrow A^{*-1}
$$

by $d \phi^{1}=\phi^{0} d^{1}$.

Assume by induction that we have constructed morphisms

$$
d^{r}: C^{*, *} \rightarrow C^{*+r, *-r+1} \text { and } \phi^{r}: C^{-r, *} \rightarrow A^{*-r}
$$

for $0 \leq r \leq n$ (with $d^{0}=0$ ) such that

$$
\sum_{k+\ell \leq n+1} d^{k} d^{\ell}=0 \text { and } d \phi^{(n)}=\phi^{(n-1)} d^{(n)} \text { on } C_{(-n)}
$$

where $d^{(n)}=\sum_{1 \leq r \leq n} d^{r}$ and $\phi^{(n)}=\sum_{0 \leq r \leq n} \phi^{r}: C_{(-n)} \rightarrow A$.

Consider the composition $\phi^{(n)} d^{(n)}: C^{-n-1, *} \rightarrow A^{*-n}$. Clearly,

$$
\phi^{(n)} d^{(n)}: C^{-n-1, *} \rightarrow Z A^{*-n}\left(\hookrightarrow A^{*-n}\right) .
$$

Form the composition $\nu \phi^{(n)} d^{(n)}: C^{-n-1, *} \rightarrow H^{*-n}(A)$ to obtain a morphism $d^{n+1}$ : $C^{-n-1, *} \rightarrow C^{0, *-n}$ such that $\rho^{0} d^{n+1}=-\nu \phi^{(n)} d^{(n)}$. Since $H^{i}\left(C^{i, *}, d^{1}\right)=0$ for $i<0$, we can extend $d^{n+1}$ on $C^{*, *}$ with $\sum_{k+\ell \leq n+2} d^{k} d^{\ell}=0$. Then $\nu \phi^{(n)} d^{(n+1)}=0$, and there is a morphism $\phi^{n+1}: C^{-n-1, *} \rightarrow \bar{A}^{*-n-1}$ with $d \phi^{(n+1)}=\phi^{(n)} d^{(n+1)}$.

Define

$$
d^{*}=\sum_{r \geq 1} d^{r} \text { and } \phi=\sum_{r \geq 0} \phi^{r}
$$

to obtain the homological resolution $\phi:\left(\operatorname{Tot}(C), d^{*}\right) \rightarrow(A, d)$. 
In particular, one can take $d^{r}=0, r \geq 2$, on $C^{*, *}$ and $\phi=\rho$ when $d_{A}=0$.

Note that in the abelian category of modules homological multicomplex resolutions were in fact constructed in [2], 3] (compare [7]).

3.1. Proof of Proposition 2, As above the proof uses the induction on the resolution degree of the projective multicomplex $C$. First show that $f_{\#}$ is an epimorphism. Let $\bar{g}: \operatorname{Tot}(C) \rightarrow B$. Consider the restriction $\bar{g}^{0}=\left.\bar{g}\right|_{C^{0, *}}: C^{0, *} \rightarrow B^{*}$; then the induced map $\left(\bar{g}^{0}\right)^{*}: C^{0, *} \rightarrow H^{*}(B)$ factors through cocycles $\left(\bar{g}^{0}\right)^{*}: C^{0, *} \stackrel{\phi^{\prime}}{\rightarrow} Z B^{*} \stackrel{\nu}{\rightarrow}$ $H^{*}(B)$. Since $H(f)$ is an isomorphism, we can define $g^{0}: C^{0, *} \rightarrow Z A^{*} \hookrightarrow A^{*}$ such that $\left(f g^{0}\right)^{*}=\left(\bar{g}^{0}\right)^{*}: C^{0, *} \rightarrow H^{*}(B)$. Obviously, there is $s^{0}: C^{0, *} \rightarrow B^{*-1}$ with $f g^{0}-\bar{g}^{0}=d s^{0}$ and then put $\overline{\bar{g}}=\bar{g}+d s^{0}+s^{0} d$ to obtain the commutative diagram

$$
\begin{array}{cll}
C^{0, *}=\operatorname{Tot}\left(C_{(0)}\right) & \stackrel{g^{0}}{\longrightarrow} & A \\
\downarrow & & \downarrow f \\
\operatorname{Tot}(C) & \stackrel{\bar{g}}{\longrightarrow} & B .
\end{array}
$$

Assume by induction that we have constructed morphisms

$$
g^{i}: C^{-i, *} \rightarrow A^{*-i}, 0 \leq i \leq n, \text { and } \tilde{g}: \operatorname{Tot}(C) \rightarrow B
$$

such that $\tilde{g} \simeq \bar{g}$ and the following diagram

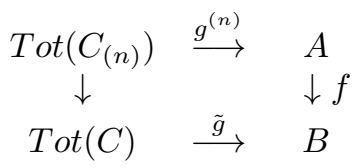

commutes. Since the above diagram is commutative and $H(f)$ is an isomorphism we can choose $g^{n+1}: C^{-n-1, *} \rightarrow A^{*-n-1}$ together with $s^{n+1}: C^{-n-1, *} \rightarrow B^{*-n-2}$ such that $d g^{n+1}=g^{(n)} d^{(n+1)}$ and $f g^{n+1}-\tilde{g}^{n+1}=d s^{n+1}$. Put $\tilde{\tilde{g}}=\tilde{g}+d s^{n+1}+s^{n+1} d$ to obtain the commutative diagram

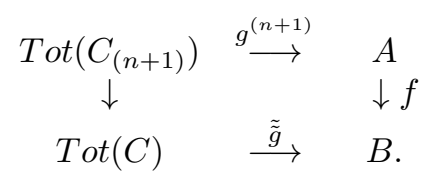

Thus, $g=\sum_{i \geq 0} g^{i}: \operatorname{Tot}(C) \rightarrow A$ is a chain map with $f g \simeq \bar{g}$, i.e. $f_{\#}[g]=[\bar{g}]$.

Now let $g, h: \operatorname{Tot}(C) \rightarrow A$ be two morphisms such that $f g$ and $f h$ are connected by a chain homotopy $s: \operatorname{Tot}(C) \rightarrow B$, i.e. $f g \simeq f h$. Clearly, $f g^{0}-f h^{0}=d s^{0}$ for $s^{0}=\left.s\right|_{C^{0, *}}$, and, since $H(f)$ is an isomorphism there is $t^{0}: C^{0, *} \rightarrow A^{*-1}$ with $g^{0}-h^{0}=d t^{0}$. Choose $t^{0}$ with $f t^{0}-s^{0}=d \beta^{0}$ for some $\beta^{0}: C^{0, *} \rightarrow B^{*-2}$. Put $h^{\prime}=h+d t^{0}+t^{0} d$ and $s^{\prime}=\left\{s^{\prime k}\right\}_{k \geq 0}$,

$$
s^{\prime k}= \begin{cases}0, & k=0 \\ s^{1}+\beta^{0} d, & k=1 \\ s^{k}, & k>1\end{cases}
$$

Then $h^{\prime 0}=g^{0}$ and $f g \underset{s^{\prime}}{\simeq} f h^{\prime}$.

Assume by induction that we have constructed a morphism $\bar{h}: \operatorname{Tot}(C) \rightarrow A$ together with chain homotopy $\bar{s}: \operatorname{Tot}(C) \rightarrow B$ such that $\bar{h}^{(n-1)}=g^{(n-1)}, \bar{h} \simeq h$ and $f g \underset{\bar{s}}{\simeq} f \bar{h}$ with $\bar{s}^{(n-1)}=0$. Since $H(f)$ is an isomorphism there is $t^{n}: C^{-n, *} \rightarrow$ 
$A^{*-n-1}$ such that $g^{n}-\bar{h}^{n}=d t^{n}$. We can choose $t^{n}$ with $f t^{n}-\bar{s}^{n}=d \beta^{n}$ for some $\beta^{n}: C^{-n, *} \rightarrow B^{*-n-2}$. Put $\overline{\bar{h}}=\bar{h}+d t^{n}+t^{n} d$ and $\overline{\bar{s}}=\left\{\overline{\bar{s}}^{k}\right\}_{k \geq 0}$,

$$
\overline{\bar{s}}^{k}= \begin{cases}0, & 0 \leq k \leq n \\ \bar{s}^{n+1}+\beta^{n} d, & k=n+1 \\ \bar{s}^{k}, & k>n+1 .\end{cases}
$$

Then $\overline{\bar{h}}^{(n+1)}=g^{(n+1)}$ and $f g \underset{\overline{\bar{s}}}{\simeq} \overline{\bar{h}}$. The induction step is completed.

Finally, we get that $g \simeq h$ as required.

3.2. Proof of Theorem 1. Apply Propositions 3 and 2 to construct the functor

$$
\varrho: K(\mathcal{A}) \rightarrow K_{\mathcal{P}}(\mathcal{A})
$$

which to each chain complex assigns its homological resolution. Again by the above propositions we deduce that $\varrho$ transforms quasi-isomorphisms into isomorphisms, so that using the universal property of the localization functor $Q$ we get the functor

$$
\bar{\varrho}: D(\mathcal{A}) \rightarrow K_{\mathcal{P}}(\mathcal{A})
$$

such that the diagram

$$
\begin{gathered}
K(\mathcal{A}) \stackrel{Q}{\longrightarrow} D(\mathcal{A}) \\
\varrho \searrow \quad \downarrow \bar{\varrho} \\
K_{\mathcal{P}}(\mathcal{A})
\end{gathered}
$$

commutes.

Now it is straightforward to check that $\varrho$ is an inverse for $\iota$.

3.3. Derived functors. Let $F: \mathcal{A} \rightarrow \mathcal{B}$ be an additive covariant functor between abelian categories with enough projectives and countable coproducts. Obviously we have the induced functor $K(F): K(\mathcal{A}) \rightarrow K(\mathcal{B})$. It is easy to verify that the composition

$$
D(\mathcal{A}) \stackrel{\bar{\varrho}}{\longrightarrow} K_{\mathcal{P}}(\mathcal{A}) \stackrel{\kappa}{\longrightarrow} K(\mathcal{A}) \stackrel{K(F)}{\longrightarrow} K(\mathcal{B}) \stackrel{Q}{\longrightarrow} D(\mathcal{B})
$$

is the left derived functor in the sense of Verdier

$$
L F: D(\mathcal{A}) \rightarrow D(\mathcal{B}) .
$$

3.4. The minimality of homological resolutions. Finally, some remarks about the minimality of homological resolutions. For example, given a chain complex $\left(H^{*}, d\right)$ on the category of modules over a principal ideal domain a homological resolution $\left(C^{*, *}, d^{*}\right) \rightarrow\left(H^{*}, d\right)$ of $\left(H^{*}, d\right)$ can be chosen to be concentrated in the resolution degrees 0 and -1 with $d^{r}=0$ unless $r=1$. On the other hand, additional structures on $C^{i, j}$ mentioned in the introduction may impose $i<-1$ (cf. [13]): Namely, if $H^{*}=\mathbb{Z}\left[x_{1}, \ldots, x_{n}\right], n>1$, is a polynomial algebra with $d=0$, then the requirement that $C^{*, *}$ is endowed with a non-commutative multiplication compatible with the bigrading imposes the multiplicative generators of the minimal resolution $C^{i, j}$ to be concentrated in resolution degrees $i \geq-n+1$, while the resolution lengthes of groups $C^{*, j}$ for $j \geq 0$ are unbounded. If one introduces a non-commutative operation $\smile_{1}$ on $C^{*, *}$ that measures the non-commutativity of the above multiplication and is compatible with the bigrading, then even the multiplicative generators can not be no longer chosen to be bounded by the resolution degree and so on. 


\section{REFERENCES}

[1] T. L. Alonso, A. L. Jeremias and M. J. Souto, Localization in categories of complexes and unbounded resolutions, Canad. J. Math., 52 (2000), 225-247.

[2] N. Berikashvili, On the differentials of spectral sequences (Russian), Proc. Tbilisi Mat. Inst., 51 (1976), 1-105.

[3] - , Zur Homologietheorie der Faserungen I, Preprintserie der Universität Heidelberg (1988).

[4] A. Dold, Zur Homotopietheorie der Kettenkomplexe, Math. Ann., 140 (1960), 278-298.

[5] S.I. Gelfand and Yu.I. Manin, Methods of homological algebra. Volume 1: Introduction to cohomology theory and derived categories (Russian), Moscow (1986).

[6] S. Halperin and J. D. Stasheff, Obstructions to homotopy equivalences, Adv. in Math., 32 (1979), 233-279.

[7] A. Heller, Homological resolutions of complexes with operators, Ann. Math., 60 (1954), 283303.

[8] M. Hovey, Model categories, Mathematical Surveys and Monographs, 63, American Mathematical Society, Providence, RI, 1999.

[9] J. Huebschmann, Minimal free multi-models for chain algebras, Georgian Math. J., 11 (2004), 733-752.

[10] B. Iversen, Cohomology of sheaves, Springer-Verlag (1986).

[11] J.P. Meyer, Acyclic models for multicomplexes, Duke Math. J., 45 (1978), 76-85.

[12] S. Saneblidze, Perturbation and obstruction theories in fibre spaces, Proc. A. Razmadze Math. Inst., 111 (1994), 1-106.

[13] - Filtered Hirsch algebras, preprint, AT/0707.2165.

[14] N. Spaltenstein, Resolutions of unbounded complexes, Compositio Math., 65 (1988), 121-154.

[15] J.-L. Verdier, Categories derivées. SGA $4 \frac{1}{2}$, Lecture Notes in Math., 568, Springer-Verlag (1976).

[16] $\longrightarrow$ Des categories derivées des categories abeliennes. Asterisque, 239 (1996).

A. Razmadze Mathematical Institute, Department of Geometry and Topology, M. Aleksidze St., 1, 0193 Tbilisi, Georgia

E-mail address: sane@rmi.acnet.ge 